\title{
Documentos sangrientos de la guerra civil española: Mina de Valdihuelo, Badajoz
}

\author{
Bloody Documents from de Spanish Civil War: Valdihuelo's mine, Badajoz, Spain
}

$M^{\mathrm{a}}$ del Rosario Fernández Falero (1), Ma Antonia Hurtado Guapo (2), Diego Peral Pacheco (3)

Facultad de Biblioteconomía y Documentación de la Universidad de Extremadura, Plazuela de lbn Marwan s/n 06071 Badajoz, rferfal@alcazaba.unex.es. (2) Servicio de Informática de la Universidad de Extremadura, Avda. de Elvas s/n 06071 Badajoz, mahurgua@alcazaba.unex.es. (3) Facultad de Medicina.dperal@alcazaba.unex.es

\begin{abstract}
Resumen
Una de las labores a desarrollar dentro de los trabajos de recuperación de la memoria histórica de la guerra civil española ha sido la de localización de documentación referida a los enterramientos de victimas de la contienda. En la mina de Valdihuelo en San Vicente de Alcántara (Badajoz) se han realizado unas excavaciones arqueológicas, resultado de las cuales ha sido la recuperación de restos humanos que se están analizando en este momento, confirmando que estos enterramientos corresponden a fallecidos durante la guerra civil e inmediatamente después. Una vez localizado el enterramiento se han realizado una serie de labores de localización de documentación con información necesaria para la identificación de las victimas, causas de su fallecimiento, etc., cuya metodología y resultados se presentan.
\end{abstract}

Palabras clave: Documentos sangrientos. Guerra Civil. Badajoz (España). Valdihuelo (España).

\section{Introducción}

En los últimos años hemos visto como ha ido aumentando el interés por investigar los hechos ocurridos durante y después de la guerra civil porque, como indica Ermengol Gassiot (Gassiot, 2005), "la recuperación de la memoria histórica ofrece una base para repensar el presente". Estos estudios se están realizando a todos los niveles. Así, no hay que olvidar que la dimensión documental del estudio de la memoria histórica en nuestro país tiene una gran relevancia debido a que la información sobre las fosas comunes son en la mayoría de los casos orales o, en el mejor de los casos, existe documentación al respecto, pero con información parcial de los hechos, a menudo sesgada o con numerosas contradicciones y a veces silenciada.

Los objetivos que se plantean en un estudio documental de este tipo son, como indica Armengol Gassiot, varios:

\begin{abstract}
To recover the historical memory of the Spanish civil war, the investigator has to locate documentation referred to the graves of victims of the fight, with information about the places such as, number of victims, cause of the death, etc. As a result of an archaeological excavation in the mine of Valdihuelo in San Vicente of Alcántara (Badajoz), human rests have been recovered that are being analyzed at this moment. However, these analyses have confirmed that these graves correspond to deceases produced during the Civil War and immediately later. The next job was the location of documentation with necessary information for the identification of the victims and causes of their death, etc. The aim is to present a methodology of documental work for these purposes and the results of this investigation.
\end{abstract}

Keywords: Bloody documents. Spanish Civil War. Badajoz (Spain). Valdihuelo (Spain).

1. Ámbito del análisis histórico general. Dentro de él y desde el punto de vista documental, se procederá a la búsqueda de datos, entre ellos el de la recuperación de la memoria oral.

2. Ámbito del análisis histórico concreto. Mediante la recuperación de datos de archivos y la memoria oral y junto al resto de informes de la investigación, se tratará de establecer cualitativa y cuantitativamente los hechos acontecidos, así como la recuperación de los cuerpos, restitución de la memoria de las víctimas y el retorno a sus familiares vivos. Finalmente, el conjunto de datos obtenidos ayuda a establecer los hechos ocurridos.

3. Ámbito de formación. La colaboración entre los distintos grupos de investigación, ayuda a la formación de investigadores especializados en estos temas; asimismo se pretende acabar con el espejismo del $A D N$ según el cual sólo mediante estudios genéticos se puede establecer 
la identidad de las víctimas y se pueden aplicar en todos los casos.

Cómo indica el mensaje de IWETEL de Genaro Luis García López, (IWETEL, 2006) hay una serie de documentos que forman parte de la memoria histórica de este país, donde se puede localizar información sobre hechos sangrientos ocurridos durante la guerra civil española, usando el término documentos sangrientos para hacer referencia a los mismos. Así titulamos este trabajo "documentos sangrientos" pues trata del estudio realizado en el Registro Civil que se encuentra en el juzgado de Paz y el Archivo Municipal de San Vicente de Alcántara, para localizar toda la información referente a personas que fallecieron el la mina de Valdihuelo o Cuarto de Abajo situada en el término municipal de San Vicente de Alcántara.

Para la realización de este trabajo recurrimos a una serie de fuentes de información para la localización de documentos referidos a estos episodios de la guerra civil (González, 1996). El problema que se nos presenta es saber si los resultados son fiables, es decir, si los datos suministrados por las fuentes de información están de acuerdo con los hechos, y como bien indica Félix González Marzo, se debe realizar una crítica de la fuente de información, valorando en primer lugar la autenticidad y en segundo lugar estableciendo la fiabilidad de la misma. El examen de fiabilidad en este caso conduce a "la confrontación de esas fuentes con las fuentes orales que constituyen la memoria viva de las personas que vivieron la guerra". La búsqueda de estos datos concretos sobre la Guerra Civil nos llevará a una serie de fuentes que no forman parte de las clásicas utilizadas para este tipo de información, tales como la "Sección de Guerra Civil" del Archivo Histórico Nacional. Puesto que se trata de un tema muy vinculado a la situación geográfica del mismo, recurriremos a las fuentes de información locales.

Este trabajo documental forma parte de un proyecto sobre recuperación de la memoria histórica en Extremadura, gracias al cual se están realizando excavaciones arqueológicas en los lugares donde se sabe que hubo enterramientos de fallecidos durante la contienda. Los restos encontrados son analizados por los especialistas en la materia y los documentalistas nos dedicamos a la localización de información archivada sobre este tema.

\section{Material y método}

Se realizaron visitas al Archivo Municipal de San Vicente de Alcántara y al Registro Civil que se encuentra en el juzgado de Paz.

Se partió de una lista de nombres recogida por miembros del equipo de arqueología, donde se encontraban algunos nombres de personas desaparecidas, y se iniciaron las búsquedas en el registro civil, con la causa, la fecha de muerte y lugar de enterramiento (Tabla I).

\begin{tabular}{|c|c|c|c|c|}
\hline Tomo & $N^{\circ}$ personas & Causa muerte & Lugar fallecimiento & Lugar enterramiento \\
\hline 58 & 14 & Movimiento Nacional (14) & San Vicente (14) & Sin especificar \\
\hline \multirow[t]{3}{*}{59} & \multirow[t]{3}{*}{9} & Movimiento Nacional (5) & San Vicente (6) & \multirow[t]{3}{*}{ Sin especificar } \\
\hline & & Lucha nacional contra el marxismo (2) & Sin especificar (1) & \\
\hline & & No consecuencia (2) & No enterrado (2) & \\
\hline \multirow[t]{2}{*}{60} & \multirow[t]{2}{*}{2} & Neumonía (1) & \multirow[t]{2}{*}{ Sin especificar (2) } & \\
\hline & & Sin especificar (1) & & \\
\hline \multirow[t]{7}{*}{65} & \multirow[t]{7}{*}{16} & Guerra (8) & Finca Marianes (1) & Cementerio (11) \\
\hline & & Fusilamiento Guerra Civil (8) & San Vicente (3) & Cuarto abajo (1) \\
\hline & & & Cementerio (5) & Puerto Hélice (1) \\
\hline & & & El Convento (1) & Finca Marianes (1) \\
\hline & & & Puerto Élice (1) & Fosa común cementerio (1) \\
\hline & & & Cuarto Abajo (1) & Cementerio/inhumado (1) \\
\hline & & & Sin especificar (4) & \\
\hline \multirow[t]{3}{*}{66} & \multirow[t]{3}{*}{3} & Fusilamiento guerra civil (1) & Sin especificar (1) & Cementerio (2) \\
\hline & & Actos bélicos guerra (1) & San Vicente (2) & Sin especificar (1) \\
\hline & & Circunstancias guerra (1) & & \\
\hline Total & \multicolumn{4}{|c|}{44 personas } \\
\hline
\end{tabular}

Tabla I. No de personas fallecidas, causa del fallecimiento y lugares de fallecimiento y enterramiento. 
La lista de partida era resultado de la búsqueda sobre personas que murieron a consecuencia de la guerra, en los tomos 65 y 66 de las Actas de Defunción, y se amplió desde el 57 al 64 . Se buscó primero el nombre de la persona que ponía en la lista resultante de los tomos 65 y 66 ; después se buscó por año de muerte (19361937), por causa de muerte y por lugar (entre éstos se dio preferencia a Valdihuelo o Cuarto Abajo). Como sabemos, puede que haya alguna persona que aunque muerta en un lugar esté inscrita en el acta de defunción como que murió en otro sitio (recordemos que en esa época se falsificaron muchos documentos)

Una vez terminado el registro civil se pasó al archivo municipal, pero estaba en trámite de organización y no se pudo encontrar nada, aunque fue posible revisar las rectificaciones al padrón. En ellas encontramos que en los años 1979-1980 se concedieron ayudas para aquellas personas que tenían o habían tenido algún familiar muerto en la guerra (Ley, 1979). Para recibir esas ayudas era necesario que los familiares de las personas fallecidas durante la guerra firmasen unos documentos, donde se especificaba que su familiar estaba muerto y poner la causa de la muerte y el lugar. Se han obtenido algunos nombres, de estos pocos documentos. Alguno aparece como fusilado o simplemente muerto en San Vicente.

También se ha mirado en el Archivo Municipal el libro de enterramientos desde el 1 de enero de 1934 hasta el 31 diciembre de 1978 y no se encontró nada. Pero se comprobó que algunas personas tenían dos nombres y en un sitio estaban localizados por uno de ellos y en otro sitio por el otro.

\section{Resultados}

Los datos encontrados en la búsqueda realizada en el registro civil en los tomos del 58-66, hacen referencia a personas que fallecieron por los epígrafes: Movimiento Nacional, Lucha nacional contra el marxismo, Fusilamiento Guerra Civil, Actos bélicos guerra, Circunstancias guerra. Se muestran en la tabla (Tabla I), en la cual se indica el número del tomo, el número de personas fallecidas inscritas en ese tomo, lugar de fallecimiento y lugar de enterramiento. En total se localizan 44 personas, que podemos considerar que fallecen durante la guerra civil y debido a ella. En la tabla II se observa la relación entra causas de la muerte y el número de personas fallecidas durante la guerra. Cómo puede verse, se aprecia con claridad que el mayor número de personas fallecidas se debe al movimiento nacional, un $44 \%$, seguidas de un $20 \%$ por fusila- miento. En la tabla III se muestra la relación entre el número de personas fallecidas y el lugar de fallecimiento.

\begin{tabular}{lc}
\hline Causas de la muerte & $N^{\circ}$ de personas \\
\hline Movimiento Nacional & 19 \\
Fusilamiento guerra civil & 9 \\
Guerra & 8 \\
Sin especificar & 3 \\
Lucha Nacional contra el & 2 \\
marxismo & \\
Actos bélicos guerra civil & 1 \\
Circunstancias guerra & 1 \\
Neumonía & 1 \\
\hline Total & 44 \\
\hline
\end{tabular}

Tabla II. Número de personas fallecidas durante la guerra y causa de la muerte.

\begin{tabular}{lc}
\hline Lugar de fallecimiento & $N^{\circ}$ de personas \\
\hline San Vicente & 25 \\
Sin especificar & 8 \\
Cementerio & 5 \\
No enterrado & 2 \\
Finca Marianes & 1 \\
El Convento & 1 \\
Puerto Élice & 1 \\
Cuarto abajo & 1 \\
\hline Total & 44 \\
\hline
\end{tabular}

Tabla III. Número de personas fallecidas durante la guerra y lugar del fallecimiento.

Puede verse con claridad que en el lugar que estamos estudiando, Cuarto Abajo o Mina de Valdihuelo, tan sólo aparece 1 fallecido $(2,27 \%)$ pero en cambio hay 8 sin especificar $(56,82 \%)$.

\begin{tabular}{lc}
\hline Lugar de enterramiento & $N^{\circ}$ de personas \\
\hline Sin especificar & 26 \\
Cementerio & 13 \\
Cementerio/Inhumado & 1 \\
Cuarto abajo & 1 \\
Finca Marianes & 1 \\
Fosa común cementerio & 1 \\
Puerto Élice & 1 \\
\hline Total & 44 \\
\hline
\end{tabular}

Tabla IV. Número de personas fallecidas durante la guerra y lugar de enterramiento.

En la tabla IV se muestra la relación entre el número de personas fallecidas y el lugar de enterramiento, que normalmente no coincide 
con el de fallecimiento. Puede verse que, en cuanto al lugar de enterramiento, si bien sólo hay un fallecido enterrado en el Cuarto Abajo, oficialmente hay 26 fallecidos -es decir, el $59,09 \%$ - para los que no se especifica el lugar de enterramiento; luego es posible que los restos encontrado en la mina de Valdihuelo, pueda corresponder a alguno de ellos.

En tabla $V$ se muestra la relación entre el número de personas fallecidas y la edad a la que han fallecido.

\begin{tabular}{|c|c|}
\hline Edades & $N^{\circ}$ de personas fallecidas \\
\hline 17 & 1 \\
\hline 18 & 1 \\
\hline 19 & 1 \\
\hline 20 & 1 \\
\hline 24 & 2 \\
\hline 25 & 2 \\
\hline 26 & 2 \\
\hline 27 & 1 \\
\hline 30 & 1 \\
\hline 31 & 3 \\
\hline 32 & 2 \\
\hline 33 & 2 \\
\hline 34 & 1 \\
\hline 35 & 2 \\
\hline 37 & 2 \\
\hline 39 & 2 \\
\hline 41 & 1 \\
\hline 42 & 3 \\
\hline 43 & 1 \\
\hline 44 & 3 \\
\hline 47 & 1 \\
\hline 49 & 2 \\
\hline 52 & 1 \\
\hline 53 & 1 \\
\hline 54 & 3 \\
\hline 61 & 1 \\
\hline Sin especificar & 1 \\
\hline Total & 44 \\
\hline
\end{tabular}

Tabla V. Relación entre el $n^{\circ}$ de personas fallecidas durante la guerra y edad de las mismas.

Podemos comprobar que la media de edad de los fallecidos por la guerra es de 34,6 , es decir entre 34 y 35 años.

En cuanto a la edad de los fallecidos, descubrimos que entre los restos encontrados en la mina se hallaron los de varios niños. Por ello, decidimos realizar una revisión de la búsqueda para ver si aparecían datos sobre niños fallecidos a causa de la guerra, buscando en las Actas de Defunción y el Libro de Enterramiento desde el 1 de enero de 1934 y el 31 de diciembre de 1940 (ya que posteriormente podía aparecer algún niño que no hubiese sido inscrito en los libros en esas fechas). Los datos localizados pueden verse en la figura 1, donde se muestra una relación del número de niños fallecidos desde el año 1936 hasta 1940. Normalmente aparecen el nombre y los dos apellidos, aunque en ocasiones sólo ponía un bebe o niño pequeño pero no especificaba nada más -esos casos no se han contabilizado en esta ocasión.

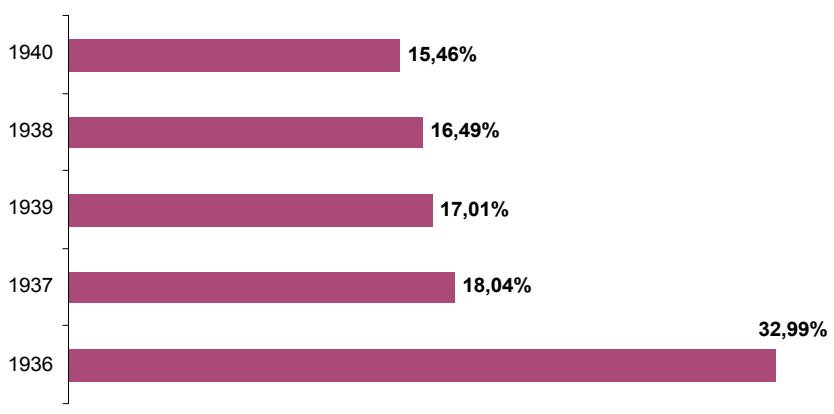

Figura 1. Relación entre el $n^{\circ}$ de niños fallecidos durante la guerra y año del fallecimiento.

Se muestra con claridad que el mayor número de niños, 32,99\% fallecen en el año 36; luego es posible que la causa de defunción de alguno de ellos sea debida directamente a la guerra.

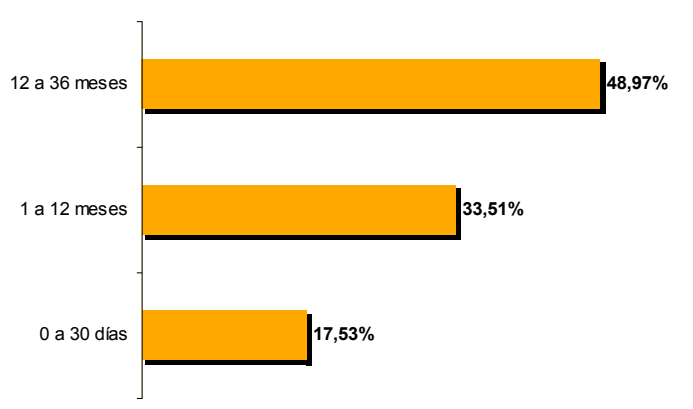

Figura 2. Relación entre el $n^{\circ}$ de niños fallecidos durante la guerra y edad de los mismos.

En lo que a la edad de los niños se refiere, se han contabilizado aquellos que habían muerto y tenían entre cero días a 36 meses. Como se indica en la figura 2, el rango de edades de los niños fallecidos está comprendido entre 1 y 3 años.

Las causas de fallecimiento muestran siempre alguna enfermedad en concreto; en ningún caso hace referencia a fusilamiento o causa de guerra, etc. De hecho, en la tabla VI se muestran 
solamente las que causaron mayor número de fallecidos (debido a la extensión de la lista).

\begin{tabular}{lc}
\hline Causa de la muerte & $N^{\circ}$ de niños fallecidos \\
\hline Gastroenteritis & 16 \\
Asistolia & 10 \\
Septicemia & 8 \\
Bronconeumonía & 8 \\
Debilidad orgánica & 7 \\
Bronquitis & 7 \\
Debilidad congénita & 6 \\
Meningitis & 5 \\
Bronconeumonía & 4 \\
aguda & \\
Gastroenteritis aguda & 3 \\
Otras & 120 \\
\hline Total & 194 \\
\hline
\end{tabular}

Tabla VI. Relación entre el número de niños fallecidos y causas de la muerte.

Finalmente al buscar el lugar de enterramiento de los niños el resultado es literalmente en "Tierra o Panteón", pero no hay referencia a ningún lugar concreto como, Cuarto Abajo, Puerto Élice, etc. Tan sólo aparecen 9 niños en los que no se especifica el lugar de enterramiento.

\section{Conclusiones}

Se han localizado 44 personas fallecidas que se pueden considerar debidas directamente a la guerra civil, pues, aunque para una de ellas figura como causa de su muerte la neumonía, no se indica ni el lugar de fallecimiento ni el lugar de enterramiento, razón por la cual decidimos incluirla en este recuento.

Respecto al Cuarto Abajo, este lugar aparece en dos ocasiones indicando a dos personas cuya causa de la muerte fue el fusilamiento, pero sólo una de ellas se entierra allí, mientras que la otra pese a ser fusilada allí, es enterrada en el cementerio.

El hecho de que en el caso de 8 personas no se especifique el lugar de enterramiento nos hace pensar que puedan haber sido enterradas en la mina.

La media de edad de las personas fallecidas es de entre 34 y 35 años, la menor es de 17 y la mayor de 61 años, encontrando una sin edad especificada.

En lo que a los niños se refiere,éestos no constan como fallecidos por causa o debido a la guerra, pese a que se encuentran restos de menores enterrados en la mina de Valdihuelo o Cuarto Abajo.

\section{Referencias}

Espinosa, F. (2003). La columna de la muerte: el avance de ejército franquista de Sevilla a Badajoz. Barcelona: Crítica, 2003.

Gassiot Ballbé, E. (2005). Arqueología forense de la Guerra Civil: justicia y memoria de la represión fascista. // Mientras tanto. 97 (2005) 95-112.

González Marzo, F. A (1996). Guerra Civil española a través de la memoria histórica depositada en los archivos. // Iber Didáctica de la Ciencia Sociales, Geografía e historia. 10 (1996) 27-36.

García López, GL (2006). Documentos sangrientos // IWETEL, lista de discusión (2006) http://listserv.rediris.es/ cgi-bin/ (Consulta: 22 enero 2007).

Ley $5 / 1979$ de 18 septiembre, sobre reconocimiento de pensiones, asistencia médico-farmacéutica y asistencia social a favor de las viudas, y demás familiares de los españoles fallecidos como consecuencia o con ocasión de la pasada guerra civil (1979). // Boletín Oficial del Estado. 233 (1979).

http://www.congreso.es/constitucion/ficheros/leyes_espa/l_0 05 1979.pdf (Consulta: 5 marzo 2007). 